\title{
Aspectos técnicos e roteiro de análise da radiografia de tórax
}

\author{
Technical aspects and analysis guide of the chest radiography \\ Danilo Tadao Wada ${ }^{1}$ (D), José Antonio Hiesinger Rodrigues ${ }^{1}$, Marcel Koenigkam Santos² (iD
}

\begin{abstract}
RESUMO
Antes de se iniciar a avaliação dos achados radiográficos no exame de radiografia (RX) de tórax, é preciso se atentar às incidências realizadas e aos parâmetros técnicos do exame. A sistematização da avaliação, utilizando a rotina semiológica e a terminologia correta, também é importante. Este artigo visa abordar os aspetos técnicos básicos para a realização de um bom exame de RX de tórax e apresentar um roteiro de avaliação sistematizada para sua correta interpretação. O exame de RX de tórax deve ser realizado, sempre que possível, com as incidências em posteroanterior (PA) e perfil. Há outras incidências que são realizadas de maneira complementar ou em situações específicas, como a anteroposterior (AP), decúbito lateral com raios horizontais (Laurel), apicolordótica, oblíquas, expiração máxima e com marcadores no tórax. Os 3 principais parâmetros técnicos do RX de tórax avaliados na rotina clínica são a dose de radiação (verificar se o RX não ficou pouco ou muito penetrado), inspiração correta (verificar se o exame não está expirado) e o alinhamento (verificar se o RX não está rodado). A sistematização na avaliação do exame de RX de tórax, seguindo uma rotina semiológica, ajuda a reduzir a chance de erros de interpretação e de se perder alterações menos importantes ou secundárias. O modelo de laudo normal pode servir de roteiro para a análise do exame.
\end{abstract}

Palavras-chave: Radiografia Torácica. Tórax. Aspectos Técnicos.

\begin{abstract}
Paying attention to the projection and technical parameters is essential before starting to interpret conventional chest radiography findings. Systematic evaluation using a semiology routine and proper terminology is important in radiography interpretation. In this article, we meant to cover the basic technical aspects for getting a good exam and propose a systematic script to correct interpretation of conventional chest radiographies. When possible, the chest radiography should be performed in both projections: posteroanterior (PA) and lateral. There are some other specific projections like anteroposterior (AP), lateral decubitus with horizontal rays (Laurel), apicolordotic, obliques, maximum expiration and radiography with external markers. The 3 main technical parameters evaluated in clinical routine are the radiation dose (related to the exposure, leading to a too brighten or too dark image), correct inspiration (verifying if the exam wasn't made in expiration) and alignment (verifying if the patient was corrected aligned to the ray direction). Systematic evaluation of conventional chest radiography going through a semiology routine helps reducing the chance of missing subtle or secondary findings. A normal exam report model could work as a guide to the exam interpretation.
\end{abstract}

Keywords: Thoracic Radiography. Chest. Technical Aspects.

1. Médico assistente da disciplina de Radiologia Torácica e Cardiovascular. Centro de Ciências das Imagens e Física Médica, Hospital das Clínicas da Faculdade de Medicina de Ribeirão Preto da Universidade de São Paulo, Ribeirão Preto (SP), Brasil.

2. Docente responsável pela disciplina de Radiologia Torácica e Cardiovascular. Fundação de Apoio ao Ensino, Pesquisa e Assistência do Hospital das Clínicas da Faculdade de Medicina de Ribeirão Preto da Universidade de São Paulo (FAEPA), Ribeirão Preto (SP), Brasil.

[ Danilo Tadao Wada. Avenida Bandeirantes, 3900 - Campus Universitário. CEP: 14049-900. Ribeirão Preto SP, Brasil. dwada@hcrp.usp.br | Recebido em: 17/02/2019 | Aprovado em: 26/04/2019 


\section{INTRODUÇÃO}

$\mathrm{O}$ exame de radiografia ( $R X)$ na avaliação do tórax representa o método de imagem mais antigo, mais barato, mais disponível ${ }^{1}$ o Departamento de In-formática do SUS (Datasus e tecnologicamente mais simples, desta maneira representando, na maioria das situações, o primeiro exame radiológico a ser solicitado para avaliação das doenças torácicas ${ }^{2}$. 0 aparelho de RX está comumente disponível mesmo nas unidades básicas de atendimento de saúde e possui ainda a vantagem de ser móvel, permitindo a realização de exames de pacientes acamados, no centro cirúrgico, centro de terapia intensiva ou nas salas de atendimento de urgência e trauma.

O RX ainda representa, apesar de todos os avanços tecnológicos dos demais métodos, o método de imagem radiológica com a maior resolução espacial (o menor pixel de imagem) ${ }^{3}$. Porém, a imagem radiográfica é uma representação bidimensional de estruturas tridimensionais, desta maneira tendo como importante desvantagem a sobreposição de estruturas na imagem, algo que não acontece com os métodos seccionais, como a tomografia computadorizada (TC) e a ressonância magnética (RM) ${ }^{4}$.

$\mathrm{Na}$ avaliação de um exame de RX de tórax, o primeiro passo consiste em identificar quais as incidências obtidas e se são suficientes para responder a questão clínica. Depois é preciso verificar se a técnica utilizada foi adequada e se não há artefatos ou deficiências que possam prejudicar a interpretação do exame ${ }^{5}$. Também se faz importante a sistematização da avaliação da imagem ${ }^{5}$, respeitando a semiologia e terminologia radiológica ${ }^{6-8}$, para que todas as informações contidas no RX sejam adequadamente identificadas, interpretadas e relatadas. A sistematização também auxilia a não deixar de descrever todos os achados na imagem, inclusive aqueles não diretamente relacionados à queixa clínica.

\section{OBJETIVOS GERAIS}

Após a leitura deste texto, o leitor deverá ser capaz de:

- conhecer as incidências básicas e complementares do RX de tórax;
- $\quad$ saber identificar problemas relacionados aos parâmetros técnicos do exame;

sistematizar a avaliação semiológica do exame.

\section{Incidências}

O exame de RX de tórax deve ser realizado, sempre que possível, com as incidências em posteroanterior (PA) e em perfil. Há outras incidências que são realizadas de maneira complementar ou em situações específicas:

- $\quad$ anteroposterior (AP)

- decúbito lateral com raios horizontais (Laurel)

- $\quad$ apicolordótica

- obliquas

- expiração máxima

- com marcadores no tórax

\section{Posteroanterior e em perfil}

Este é o protocolo padrão e deve ser realizado sempre que possível, com o paciente em ortostase (de pé) e em inspiração profunda ${ }^{4,5}$. Como a radiografia é uma representação bidimensional de uma estrutura tridimensional, a presença de duas incidências auxilia a localizar e caracterizar as alterações mais adequadamente. Devido a divergência dos feixes de raio $X$ emitidos pela ampola, a imagem em PA é preferida em relação à $A P$, pois aproxima o coração do detector e reduz sua magnificação ${ }^{5}$. A posição em PA também auxilia a retirar as escápulas dos campos pulmonares (figuras 1 e 2).

O protocolo padrão do $\mathrm{RX}$ de tórax é realizado em PA e em perfil, com o paciente de pé e realizando uma inspiração profunda.

A distância mínima do paciente para a ampola (distância foco-objeto) deve ser de 1,5 m, sendo ideal a distância tecnicamente padronizada de $1,8 \mathrm{~m}^{4,9}$. $\mathrm{O}$ paciente pode elevar os membros superiores, em geral com apoio (segurando em algo), ou apoiar as mãos na cintura afastando os ombros, além de encostar a porção anterior do tórax no detector. 


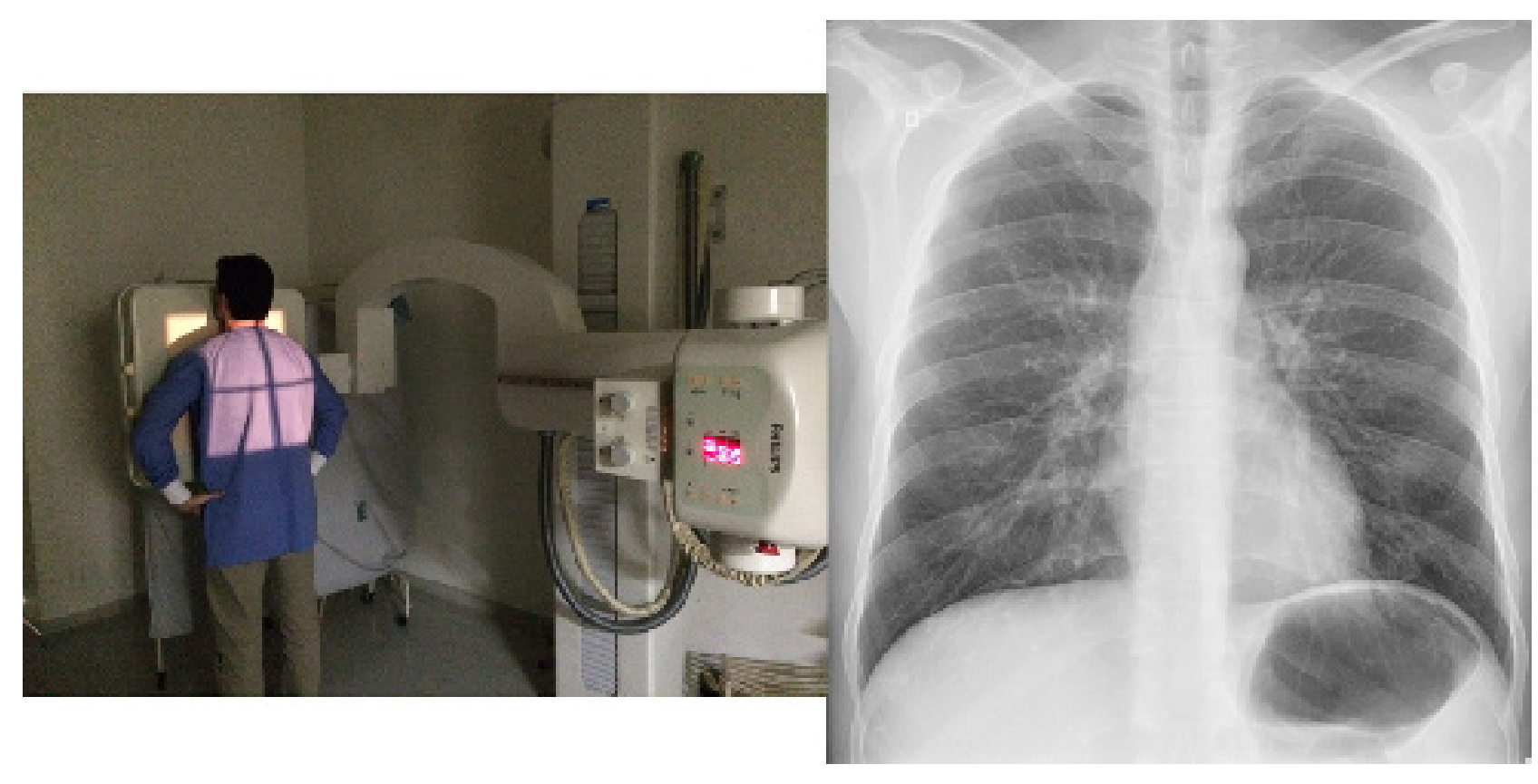

Figura 1: Posicionamento do paciente para realização da radiografia em PA do tórax e a respectiva imagem do exame.

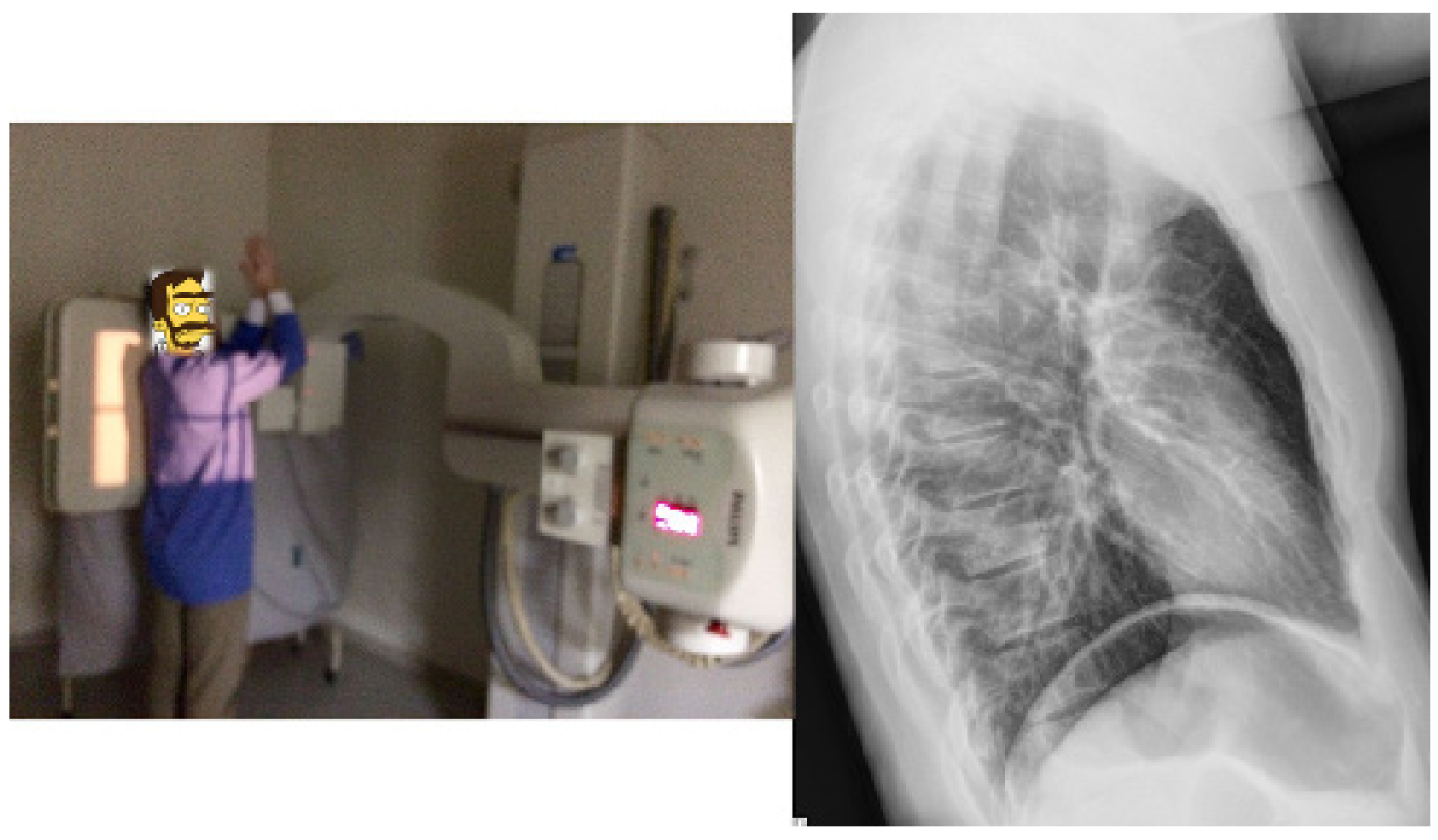

Figura 2: Posicionamento do paciente para realização da radiografia em perfil do tórax e a respectiva imagem do exame.

A imagem em perfil pode ser obtida com o lado direito ou esquerdo junto ao detector, mais comumente o segundo, para reduzir a magnifi- cação do coração (feixe de raios $X$ penetra pela direita). Quanto mais próxima a estrutura do detector radiológico, menor a ampliação e deforma- 
ção e melhor a nitidez. É comum que uma lesão localizada atrás do coração ou nas bases atrás das cúpulas diafragmáticas seja pouco visível na imagem em PA, e nestas situações a incidência em perfil é útil. O perfil também auxilia a detectar opacidades intersticiais, já que os pulmões estão sobrepostos, salientando a presença de opacidades sutis e difusas ${ }^{10}$. Por vezes, a imagem em perfil pode ser obtida após a ingestão oral de meio de contraste positivo pelo paciente, para auxiliar na avaliação do esôfago, hérnia hiatal e na dilatação do átrio esquerdo ${ }^{11-14}$.

\section{Anteroposterior}

O RX de tórax em AP é realizado quando o paciente está impossibilitado de realizar o exame em PA e em perfil, como por exemplo nas crianças pequenas, pacientes no leito, em cadeiras de roda ou com dificuldades de mobilização. Pode ser realizada com o paciente sentado ou deitado e o feixe de raios $X$ penetra por via anterior, estando a porção posterior do tórax em contato com o detector (figura 3$)^{5}$.

$\mathrm{Na}$ imagem em AP o coração encontra-se amplificado ${ }^{4}$, já que está mais afastado do detector, tornando menos fidedigna a caracterização do aumento da área cardíaca através do índice cardiotorácico. Esta incidência também dificulta a avaliação de opacidades pulmonares anteriores, como no lobo médio e língula ${ }^{5}$. Se o paciente estiver deitado, prejudicada ainda a adequada avaliação da trama vascular pulmonar, como por exemplo na caracterização de redistribuição ou cefalização relacionada à congestão pulmonar.

A imagem em AP tem pior qualidade e pode atrapalhar a avaliação da área cardíaca, trama vascular pulmonar e de opacidades pulmonares.

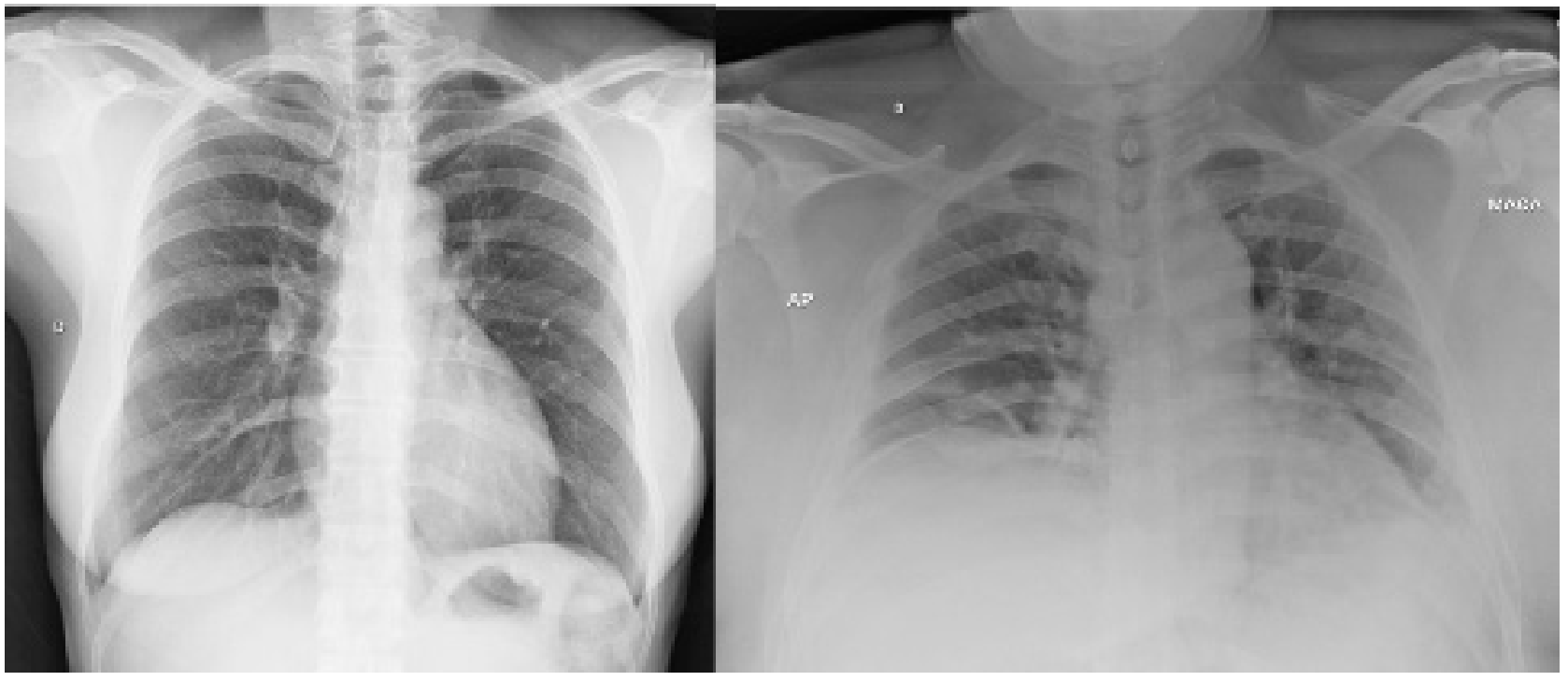

Figura 3: Radiografia do tórax em PA ortostático (esquerda) e AP deitado (direita). Notar a diferença de qualidade das imagens, sendo que a imagem em PA permite melhor avaliação dos pulmões e estruturas mediastinais.

\section{Decúbito lateral com raios horizontais (Laurel)}

Esta incidência é indicada principalmente para auxiliar na identificação de derrame pleural e na diferenciação de derrame e espessamento pleural. Deve ser realizada com o paciente deitado sobre o lado em que se suspeita haver doença pleural. Desta maneira, se há suspeição de doença pleural à direita, com obliteração do seio costofrênico direito, a imagem deve ser realizada com o paciente em decúbito lateral direito. A ampola de raios $X$ é colocada horizontalmente, com o feixe incidindo anteriormente no corpo do paciente e o detector colocado junto a face posterior do tórax (figura 4$)^{4}$. Menos comumente, o decúbito 
lateral pode ser utilizado na suspeição de pneumotórax, devendo, nesta situação, o lado suspeito estar para cima.

O decúbito lateral é utilizado principalmente para avaliação de doença pleural, identificando o líquido pleural livre.

Na presença de derrame pleural, observamos que o líquido escorre junto ao gradil costal inferior, formando um nível líquido na imagem em decúbito lateral4. É importante lembrar que o líquido que escorre está livre, portanto, por vezes, quando da presença de septações e loculações, pode-se não identificar o aspecto típico no RX em decúbito lateral ou identifica-se que apenas parte do líquido escorre, indicando tratar-se de um derrame pleural complexo. Nestes casos, é apropriado complementar a avaliação com outros métodos de imagem, como a tomografia computadorizada (TC) com uso de contraste endovenoso, ultrassonografia (US) ou a RM.

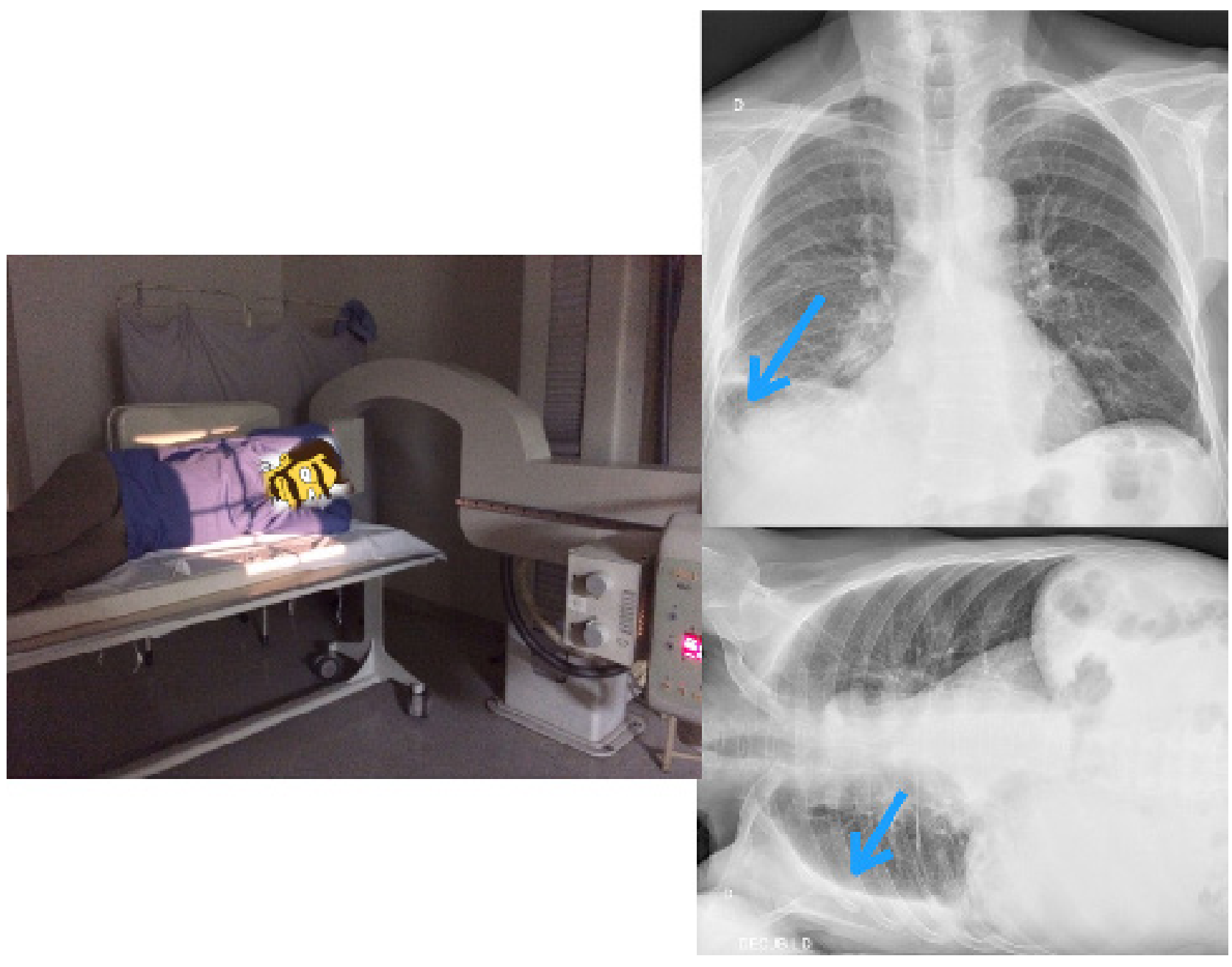

Figura 4: Posicionamento para o RX Laurel (figura esquerda). Imagens em PA (superior direita) e decúbito lateral direito com raios horizontais (inferior direita) de outro paciente, evidenciando a obliteração do seio costofrênico direito, com o derrame pleural melhor evidenciado (escorreu junto ao gradil costal) no decúbito ipsilateral (setas). 


\section{Demais incidências}

As demais incidências radiográficas são menos utilizadas atualmente, principalmente devido ao desenvolvimento e maior disponibilidade da TC de tórax.

A incidência apicolordótica é realizada com o paciente em hiperlordose, as costas apoiadas sobre o detector e o feixe penetrando pelo tórax anterior- mente ${ }^{4,9}$. Esta posição pode ser incômoda para o paciente, tendo como opção angular o tubo de RX de maneira a simular o posicionamento e obtendo assim imagem semelhante. Na imagem apicolordótica, as clavículas estão elevadas e são retiradas dos campos pulmonares, facilitando a identificação de lesões nas regiões apicais, assim como no lobo médio e língula. O coração e os grandes vasos ficam de avaliação prejudicada (figura 5).

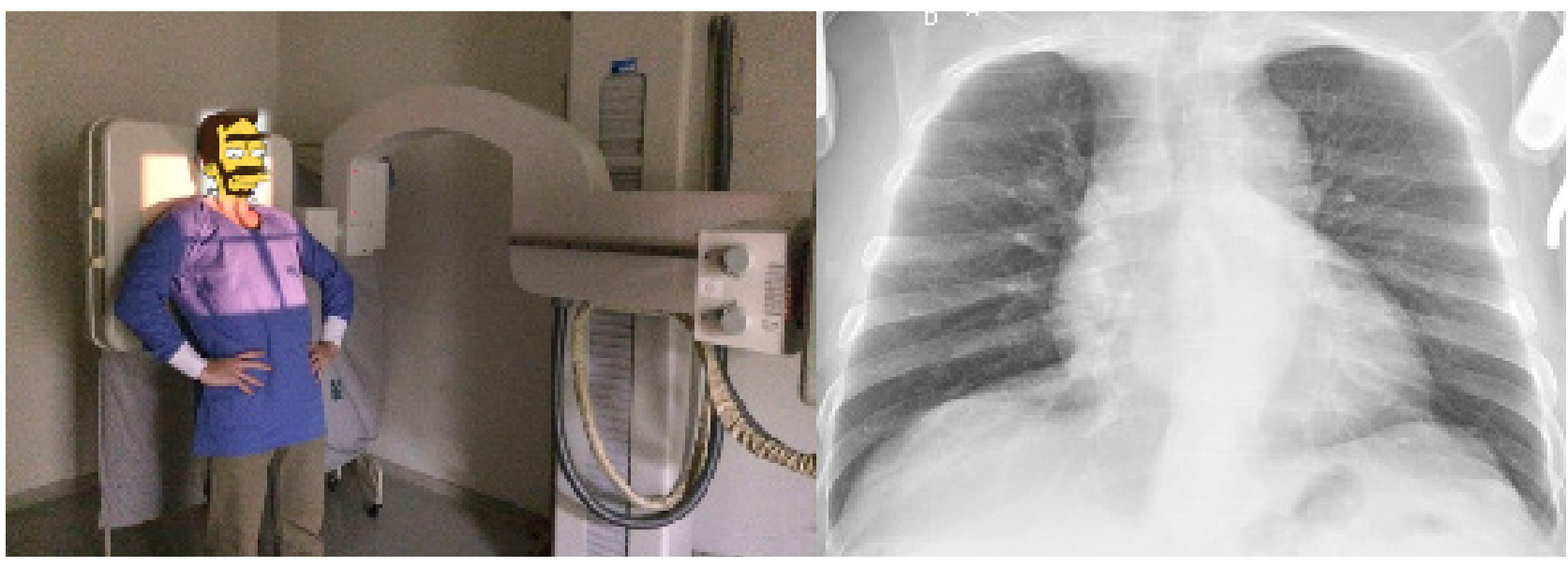

Figura 5: Posicionamento para aquisição de imagem na incidência apicolordótica (esquerda). Imagem do RX (direita) mostrando o deslocamento superior das clavículas, permitindo melhor visibilização dos ápices pulmonares.

As imagens obliquas, que podem ser obtidas tanto na incidência posteroanterior à direita quanto à esquerda ${ }^{9}$, podem auxiliar na avaliação do aumento isolado de câmaras cardíacas ou na melhor identificação de alterações parcialmente encobertas por estruturas mediastinais. Hoje estas incidências são pouco utilizadas, principalmente devido à maior disponibilidade da ecocardiografia para avaliação cardíaca.

O exame em expiração máxima pode ser obtido em PA ou AP e é utilizado para auxiliar a detectar pequeno pneumotórax, que se torna mais evidente na expiração quando comparado à imagem em inspiração ${ }^{4,5}$. A imagem em expiração pode também auxiliar no diagnóstico de aspiração de corpo estranho, identificando o aprisionamento aéreo relacionado ao mecanismo valvular do objeto na via aérea central (o lobo ou segmento pulmonar distal se mantém hipertransparente e expandido na expiração) (figura 6$)^{4,5}$.

Por vezes, os mamilos ou lesões de pele e subcutâneo (por ex.: verrucosidades) podem simu- lar nódulos ou outras opacidades pulmonares no RX de tórax. Os mamilos muito comumente são vistos como pequenas opacidades arredondadas projetadas no campo pulmonar inferior, nem sempre de maneira simétrica ${ }^{5}$. Nestes casos, podemos realizar a radiografia com um marcador torácico metálico ou radiopaco, como um clip ou pequena quantidade de meio de contraste positivo, colocado sobre o mamilo ou uma eventual lesão de pele (figura 7).

A incidência apicolordótica pode auxiliar na avaliação de lesões apicais. As incidências obliquas podem ajudar a identificar aumento isolado de câmara cardíaca. A expiração máxima ajuda a identificar pequeno pneumotórax e aspiração de corpo estranho. A imagem com marcador torácico é útil na identificação do mamilo ou de uma lesão de pele que simula nódulo pulmonar. Estas são pouco utilizadas atualmente. 


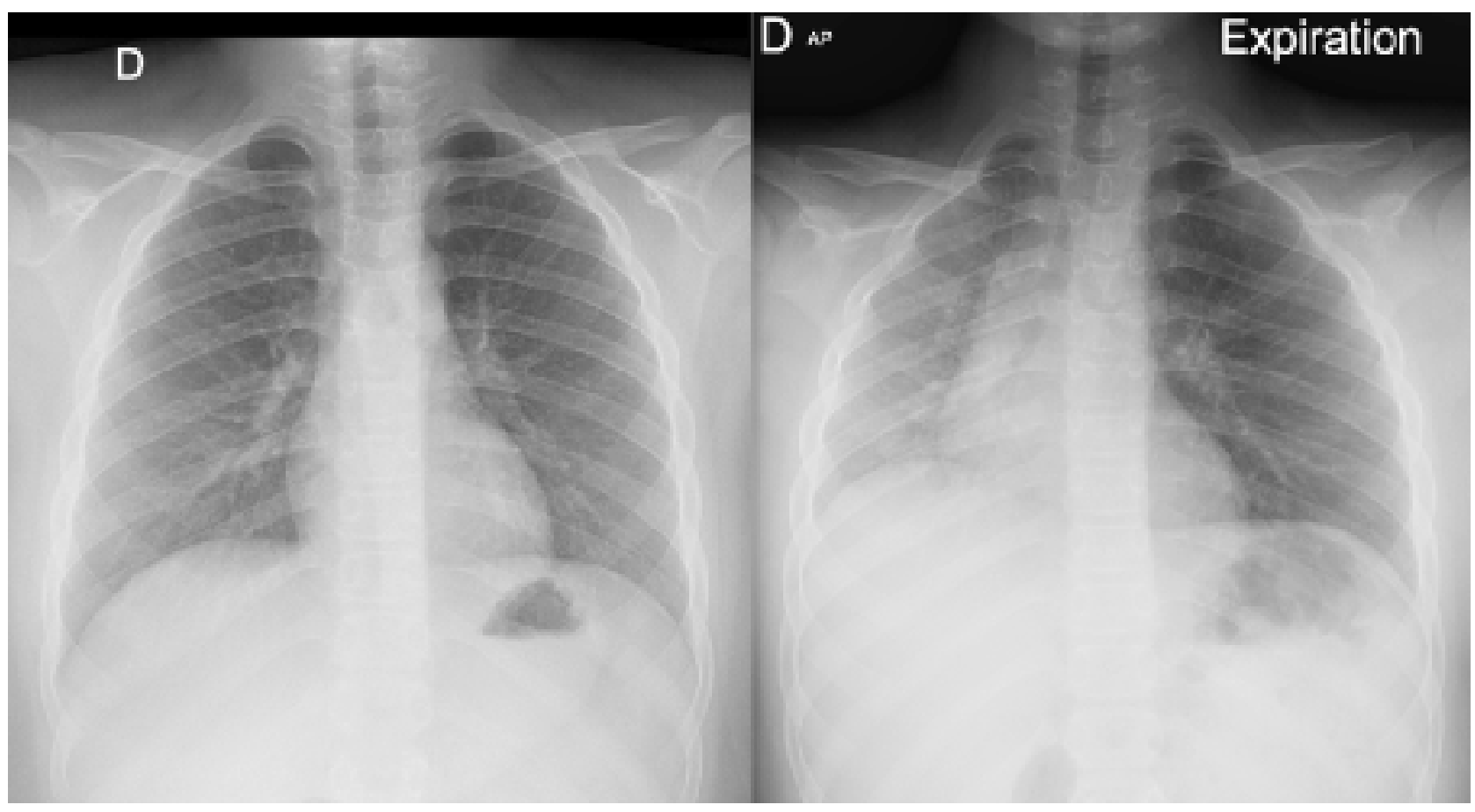

Figura 6: Imagens em inspiração (esquerda) e expiração (direita) de um paciente com corpo estranho (peça de brinquedo) impactado em brônquio fonte esquerdo. Na fase expiratória é possível identificar o aprisionamento aéreo de todo pulmão esquerdo, que não reduz de volume como o pulmão direito normal. O mediastino desvia-se à direita e a cúpula esquerda mantém-se rebaixada.

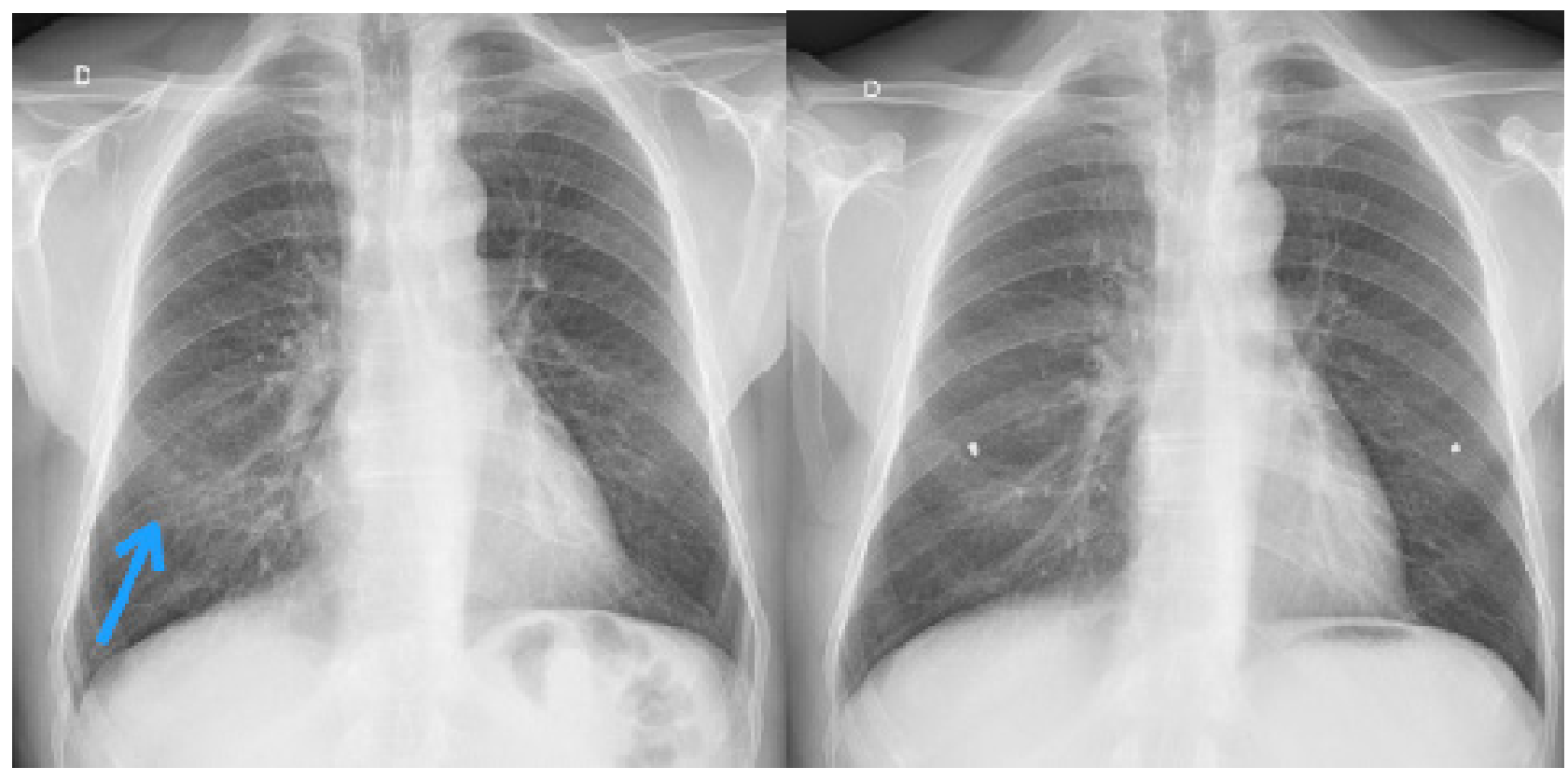

Figura 7: Radiografia PA do tórax sem e com marcadores metálicos nos mamilos. Na grafia à esquerda, observa-se a imagem do mamilo (seta) que pode simular nódulo pulmonar. 


\section{Parâmetros Técnicos}

Os 3 principais parâmetros técnicos do RX de tórax avaliados na rotina clínica são:

- $\quad$ regime de dose (penetração do $\mathrm{RX}$ )

- $\quad$ inspiração correta

- $\quad$ alinhamento (centrado ou não)

\section{Regime de dose}

Em um exame de RX de tórax, nas incidências em PA ou AP, com dose adequada e boa penetração da radiação, é possível identificar a coluna vertebral apenas nas suas porções mais superiores (T1 a T3), sendo o terço inferior da coluna obscurecido pela sombra cardíaca e do abdome superior. Quando identificamos toda a coluna, descrevemos que a grafia está muito penetrada, com dose excessiva de radiação, enquanto que, quando não se identifica nenhuma porção da coluna, descrevemos que o exame está pouco penetrado (figura 8).

Atualmente, com o advento da imagem digital, as situações de erro de regime de dose podem ser corrigidas após a realização do exame, reduzindo a necessidade de repetições e novas exposições do paciente à radiação ionizante ${ }^{5}$.

Na grafia em PA ou AP adequadamente penetrada, identificamos bem apenas o terço superior da coluna dorsal.

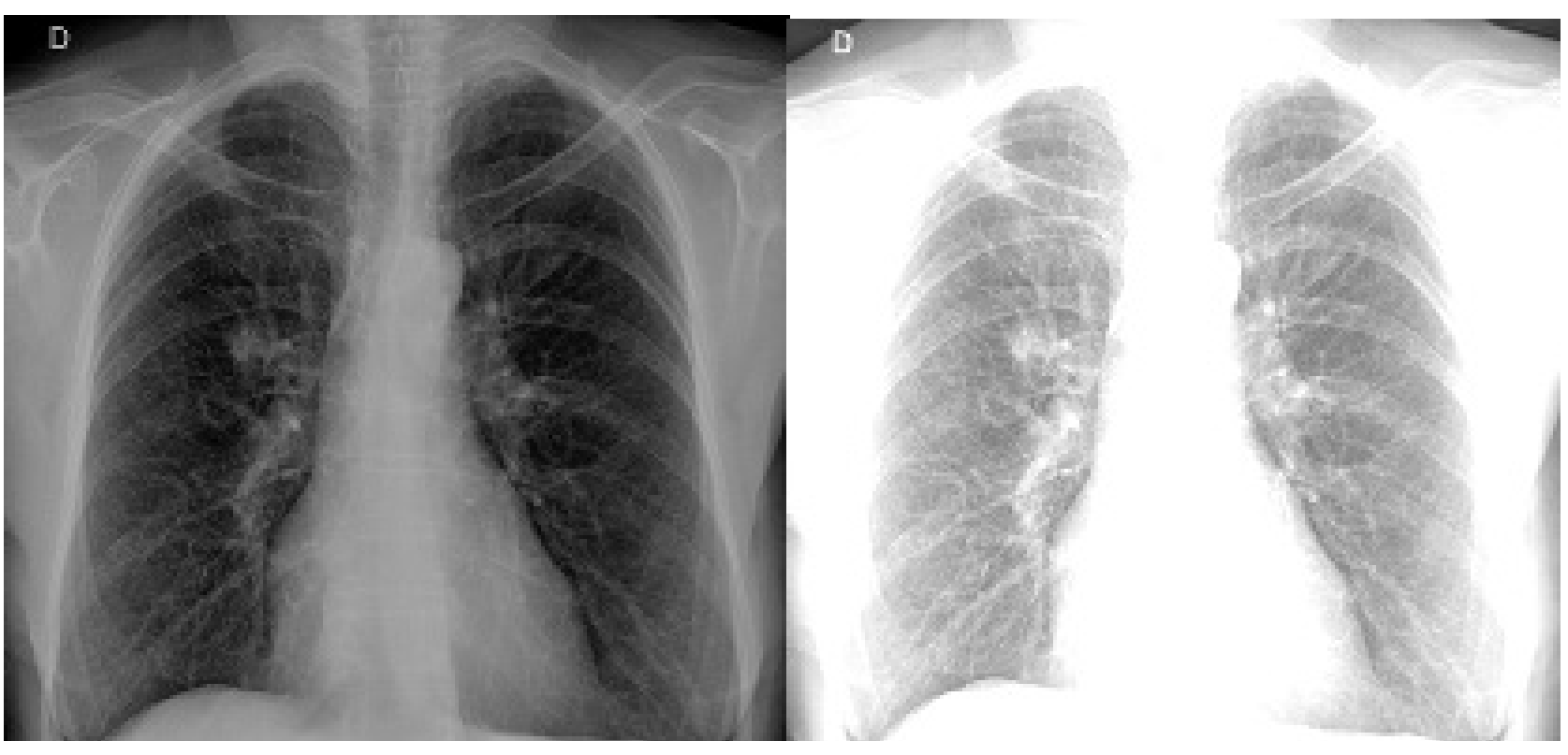

Figura 8: Exemplos de imagens obtidas com técnicas diferentes. À nossa esquerda, uma imagem muito penetrada, escura. À nossa direita, uma imagem pouco penetrada, com baixa dose de radiação.

\section{Inspiração}

Para a realização do exame de RX de tórax, o paciente é solicitado a realizar uma apneia inspiratória máxima, ou seja, "encher bem o peito de ar e segurar". Nos exames realizados com inspiração correta, devemos identificar de 9 a 11 arcos costais posteriores projetados sobre os campos pulmonares (figura 9) ${ }^{9}$.
Uma radiografia pouco inspirada pode dificultar a identificação e caracterização de opacidades pulmonares, doença pleural ou mesmo indicar uma falsa cardiomegalia ${ }^{4}$.

A correta inspiração permite identificar de 9 a 11 arcos costais posteriores sobre os pulmões. 


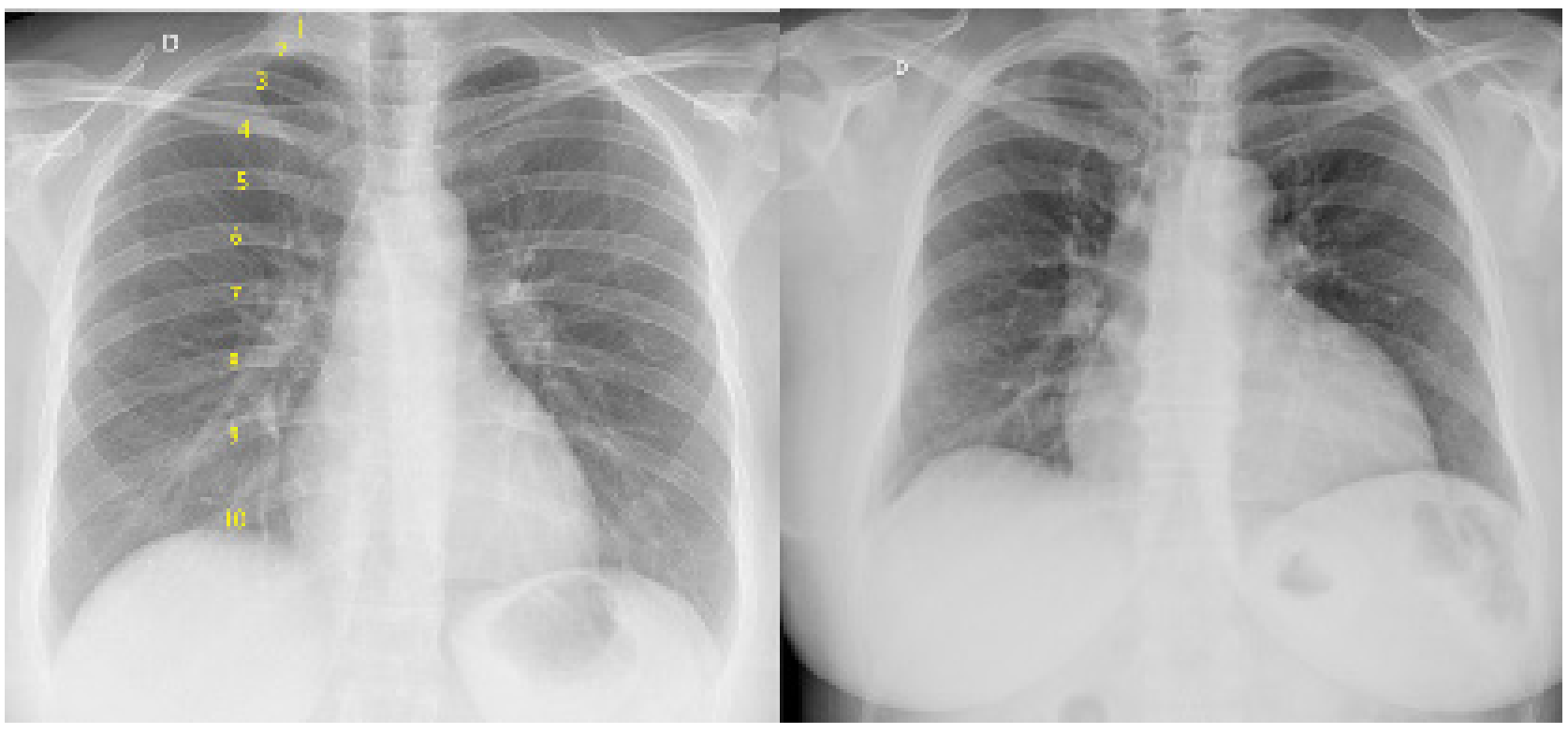

Figura 9: A imagem à esquerda mostra uma aquisição com inspiração correta, destacando a identificação de 10 arcos costais posteriores. À direita temos uma imagem pouco inspirada da mesma paciente, mostrando como há diferença do volume e transparência pulmonar e da área cardíaca.

\section{Alinhamento}

Em relação ao alinhamento do paciente, no exame centrado, as bordas mediais das clavículas têm a mesma distância para o centro da coluna dorsal (imagens em PA ou AP). Esta avaliação é mais fácil de ser realizada tendo como referência o processo espinhoso do corpo vertebral ao nível das clavículas na imagem. É importante também que as escápulas estejam projetadas fora dos campos pulmonares, de maneira simétrica, principalmente quando realizamos a aquisição em PA (figura 10).

A grafia rodada distorce a anatomia radiológica normal do tórax, podendo mascarar lesões ou mesmo criar falsas imagens que simulam alterações patológicas ${ }^{4,5}$.

Em relação ao alinhamento do detector, é importante verificar se o paciente está no centro da imagem, ou seja, a coluna é equidistante em relação às margens laterais da imagem de RX. Quando o paciente não está centrado na imagem, pode ocorrer de um hemitórax ficar mais penetrado (hipertransparente) em relação ao outro, prejudicando a avaliação correta do parênquima pulmonar ${ }^{5,9}$.
Na grafia adequadamente alinhada, as bordas mediais das clavículas são equidistantes da coluna e as escápulas estão projetadas fora dos campos pulmonares. A coluna do paciente encontra-se no centro da imagem, equidistante das margens laterais.

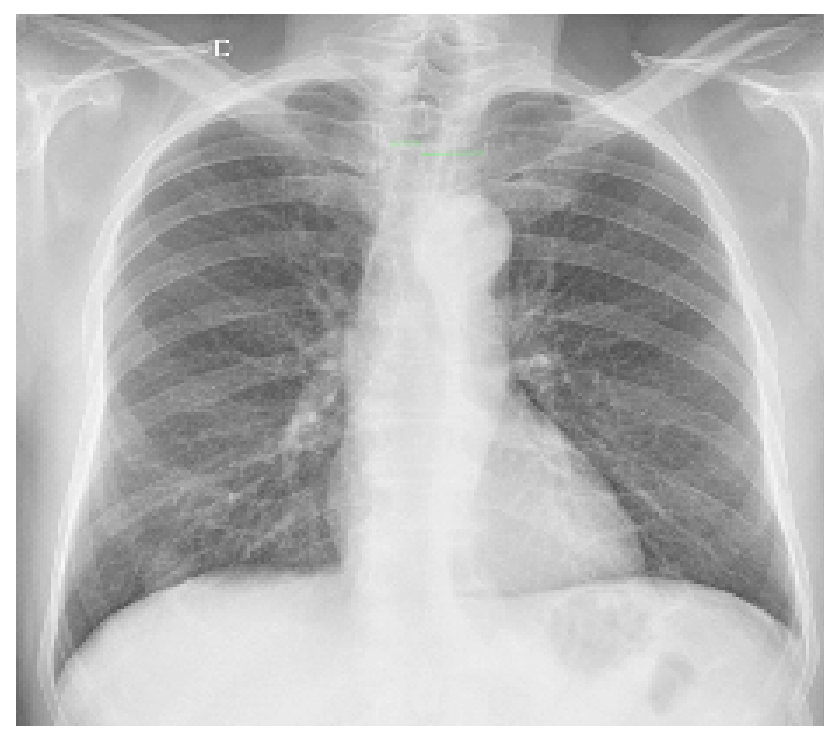

Figura 10: Exemplo de uma radiografia de tórax em PA pouco "rodada". Notar a assimetria de distância das bordas mediais das clavículas para a coluna. 


\section{Sistematização da Avaliação (Rotina Semiológica)}

A sistematização da avaliação do exame radiográfico deve ser sempre adotada, respeitando a semiologia e a terminologia radiológica, para que todas as informações contidas no RX sejam adequadamente identificadas, interpretadas e relatadas. A sistematização também auxilia a não deixar de descrever todos os achados na imagem, inclusive aqueles não diretamente relacionados à queixa clínica ou à história fornecida pelo médico solicitante do exame ${ }^{4,5}$.

- A sistematização na avaliação do exame de RX de tórax, seguindo uma rotina semiológica, ajuda a reduzir a chance de erros de interpretação e de se perder alterações menos importantes ou secundárias.

Evidentemente que não existe uma regra fixa quanto ao jeito de avaliar o exame de RX de tórax, que pode variar de acordo com o serviço médico, ou mesmo com a experiência do médico que analisa as imagens. Porém, é importante que cada médico crie sua rotina de avaliação, para que assim possa sempre identificar todas as alterações presentes na imagem.

A seguir, apresentamos uma sugestão de modelo de relatório normal, que pode atuar como guia na avaliação sistematizada do RX de tórax:

1. PARTES MOLES: sem alterações

2. ARCABOUÇO ÓSSEO: íntegro.

3. MEDIASTINO E TRAQUEIA: centrados.

4. HILOS E TRAMA VASCULAR PULMONAR: aspecto habitual.

5. PAREANQUIMA PULMONAR: transparência normal, sem opacidades.

6. CÚPULAS E SEIOS COSTOFRÊNICOS: livres.

7. CORAÇÃO: morfologia e índice cardiotorácico normais.

8. AORTA: sem alterações.

A presença de aparatos médicos, como tubos, drenos e cateteres, pode ser descrita no início do relatório.
No item 1 descrevemos as alterações das partes moles torácicas (pele, subcutâneo, músculos, mamas), como por exemplo a presença de calcificações, assimetria mamária ou enfisema. As alterações do abdome superior ou região cervical, quando identificadas no RX de tórax, podem também aqui ser descritas.

No item 2 descrevemos as alterações identificadas na coluna, arcos costais, clavículas, ossos dos ombros e demais componentes ósseos identificados. Mais comumente, identificamos alterações degenerativas e fraturas, por vezes, lesões suspeitas.

Nos itens 3 e 4 avaliamos o mediastino, a traqueia, os hilos pulmonares e a trama vascular pulmonar. Nestes itens descrevemos, por exemplo, quando há alargamento mediastinal, desvio da traqueia e estruturas mediastinais, aumento vascular ou linfonodal do hilo pulmonar, deslocamento hilar, redistribuição ou cefalização da trama vascular pulmonar.

No item 5 descrevemos a presença das opacidades pulmonares, sejam elas difusas ou focais. Aqui indicamos a presença de opacidades alveolares, intersticiais, nódulos, massas e cavidades, entre outros.

O item 6 indica a busca pelos marcadores de doença pleural (derrame, espessamento), que em geral se manifesta através da obliteração das cúpulas diafragmáticas e dos seios costofrênicos. Quando necessário, pode-se solicitar a realização da imagem em decúbito lateral com raios horizontais para complementar a avaliação.

No item 7 avaliamos a silhueta cardíaca. A área cardíaca é medida através do índice cardiotorácico (relação do maior diâmetro transverso do coração sobre o diâmetro interno da caixa torácica ao nível cardíaco), que no adulto é menor ou igual a 50\%, quando aferido na imagem em PA (inspirada e em ortostase $)^{15,16}$. Quando há alteração focal de arco cardíaco, indicando, por exemplo, aumento isolado de câmara cardíaca ou hipertensão pulmonar, também descrevemos neste item.

O último item compreende a avaliação da aorta torácica, realizada principalmente ao nível do botão aórtico, que forma o primeiro arco cardíaco à esquerda superiormente. Neste item descrevemos, por exemplo, a ectasia e ateromatose aórtica. 


\section{CONCLUSÃO}

Neste texto foram apresentados e discutidos os seguintes tópicos:

- incidências básicas e complementares de RX de tórax, como são obtidas e suas indicações

- problemas relacionados aos parâmetros técnicos do exame, como identificar e o que significam

- a sistematização da avaliação semiológica do exame, sua importância e um roteiro sugerido de análise

\section{REFERÊNCIAS}

1. Freitas MB De, Yoshimura EM. De Diagnóstico Por Imagem E Da Freqüência De Exames Radiológicos No Estado De São Paulo. Radiol Bras. 2005;38(5):347-354. doi:10.1590/S0100-39842005000500008

2. Santos JS dos, Pereira Jr GA, Bliacheriene AC, Forster AC. Protocolos Clínicos e de Regulação. Elsevier Editora Ltda; 2012.

3. Lin $E$, Alessio A. What are the basic concepts of temporal, contrast, and spatial resolution in cardiac CT? J Cardiovasc Comput Tomogr. 2009;3(6):403-408. doi:10.1016/j.jcct.2009.07.003

4. Goodman LR. Felson: Princípios de Radiologia Do Tórax: Estudo Dirigido. 2. ed. Atheneu; 2001.
5. Lacey G De, Morley S, Berman L. Radiografia Do Tórax Um Guia Prático. Elsevier Editora Ltda; 2010.

6. Hansell DM, Bankier A a, MacMahon H, McLoud TC, Müller NL, Remy J. Fleischner Society: Glossary of Terms for Thoracic Imaging. Radiology. 2008;246(3):697-722. doi:10.1148/radiol.2462070712

7. Souza Jr. AS, Araujo Neto C de, Jasinovodolinsky D, et al. Terminologia para a descrição de tomografia computadorizada do tórax: Sugestões iniciais para um consenso brasileiro. Radiol Bras. 2002;35(2):125-128. doi:10.1590/S0100-39842002000200016

8. Silva CIS, Marchiori E, Souza Júnior AS, Müller NL. Consenso brasileiro ilustrado sobre a terminologia dos descritores e padrões fundamentais da TC de tórax. J Bras Pneumol. 2010;36(1):99-123. doi:10.1590/S180637132010000100016

9. Bontrager KL, Lampignano J. Tratado de Posicionamento Radiografico E Anatomia. 7th ed. Elsevier Editora Ltda; 2010.

10. Green DB, Legasto AC, Drexler IR, Gruden JF. Pulmonary fibrosis on the lateral chest radiograph: Kerley $D$ lines revisited. Insights Imaging. 2017;8(5):483-489. doi:10.1007/s13244-017-0565-2

11. Levine MS, Rubesin SE, Laufer I. Barium Esophagography: A Study for All Seasons. Clin Gastroenterol Hepatol. $2008 ; 6(1): 11-25$. doi:10.1016/j.cgh.2007.10.029

12. Levine MS, Carucci LR. Esophageal abnormalities in gastroesophageal reflux disease. Abdom Radiol. 2018;43(6):1284-1293. doi:10.1007/s00261-017$1412-0$

13. Zoghbi WA. CARDIOVASCULAR IMAGING IN THE 21ST CENTURY. 2014;(3):2014.

14. Spindola-Franco H, Fish BG. Radiology of the Heart. Springer US; 2012. 


\section{MATERIAL SUPLEMENTAR}

\section{EXERCÍCIOS}

1. Em relação às incidências do RX de tórax, correlacione:

A. Protocolo padrão

( ) AP

B. Para avaliar derrame pleural

( ) Decúbito lateral com raios horizontais

C. Paciente acamado ou de difícil mobilização

( ) PA e em perfil

D. Suspeita de nódulo no ápice do pulmão

( ) Apicolordótica

E. Avaliação de aumento do átrio esquerdo

( ) Obliquas

2. O RX de tórax realizado em expiração máxima pode ajudar em que situações?

3. O que há de errado com esta radiografia de tórax em relação à técnica de aquisição da imagem? O que pode ser feito para melhorar?
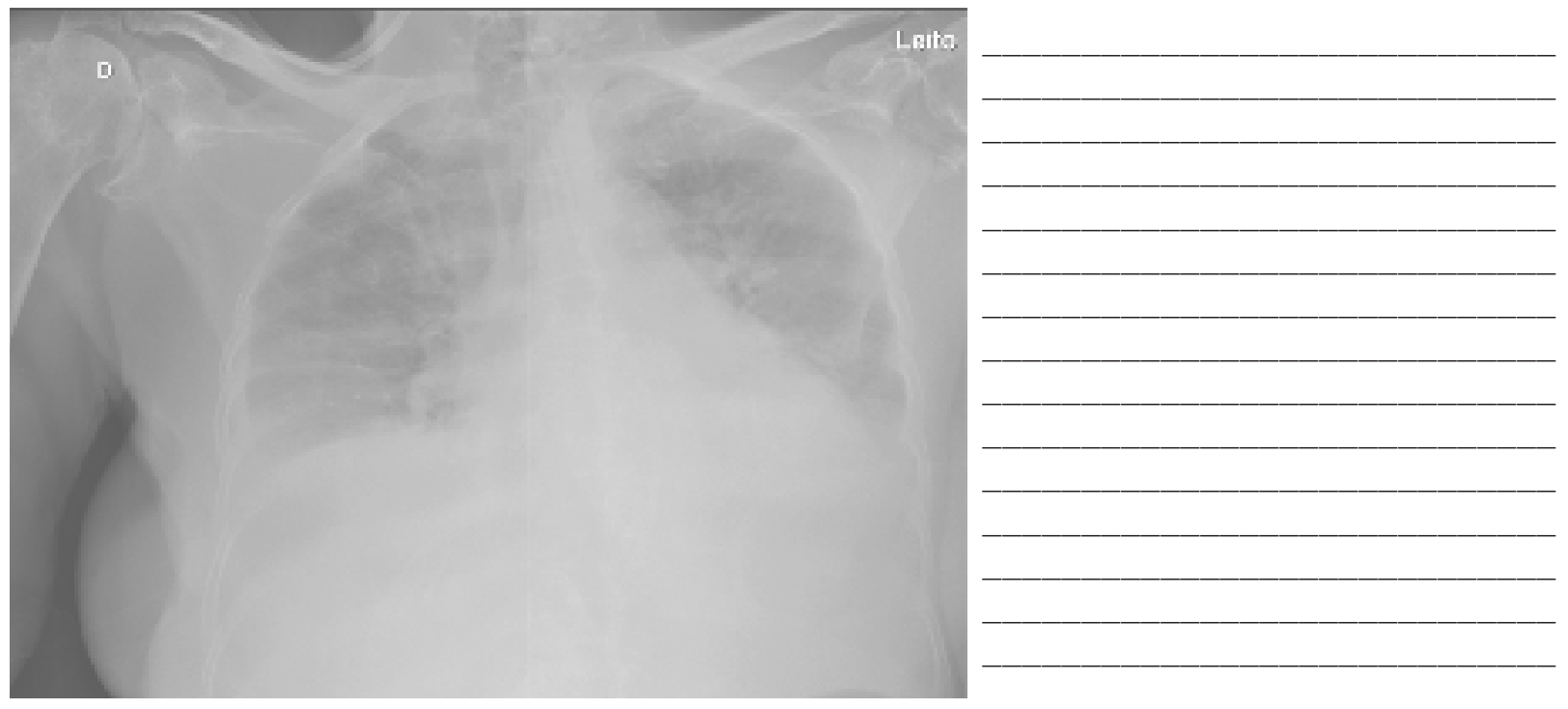

4. Como identificamos que um exame de RX de tórax realizado em PA e em perfil está adequadamente centrado?

5. Qual das alternativas abaixo não faz parte de um relatório de RX de tórax normal?

a) Estruturas ósseas íntegras

b) Trama vascular pulmonar normal

c) Ausência de opacidades pulmonares

d) Índice cardiotorácico dentro da normalidade

e) Linfonodos mediastinais de aspecto habitual 